\title{
THE ROLE OF NEIGHBORHOOD RELATIVE FREQUENCY IN SPOKEN WORD RECOGNITION
}

\author{
Philippe Mousty*, Monique Radeau*, Ronald Peereman** and Paul Bertelson* \\ *Laboratoire de Psychologie expérimentale, Université Libre de Bruxelles, Brussels, Belgium \\ **L.E.A.D./C.N.R.S, Université de Bourgogne, France
}

\begin{abstract}
According to current models of word recognition, the time to recognize a word would be affected by the frequency of its neighbors. The present study examined the role of neighborhood frequency in spoken word recognition, using a definition of the neighborhood in terms of the last candidates of the cohort aligned from onset with the input [8]. Two sets of disyllabic CVCV words were selected which differed by the relative frequency of occurrence of their more frequent neighbor (one set of words having no more frequent neighbor, and the other at least one). They were compared in three different tasks: lexical decision, repetition and gating. No effect of relative frequency was obtained either with the lexical decision task, or the repetition task. The gating task also failed to show reliable evidence for the influence of neighborhood frequency on both recognition points and isolation points (for which subjects' confidence is not taken into account). The results are discussed in terms of the nature of the competitor space of a spoken word.
\end{abstract}

\section{INTRODUCTION}

Current models of spoken word recognition assume that the selection of a lexical candidate would depend on a process of mutual differentiation between the activation levels of the target word and its competitors. This assumption predicts that a word with competitors of higher usage frequency should be recognized more slowly than a word matched on frequency but with lower frequency competitors.

Contrary to printed word research where the neighborhood is classically defined in terms of the n-count notion, which refers to the number of words that can be obtained by changing one letter of the target word [3], there is no similar consensus regarding the nature of the competitor set for a spoken word. Directional models like COHORT theory [8] consider, as competitors, neighbors belonging to the target cohort, i.e., all the lexical candidates activated by the initial sequence of the target word until its uniqueness point, i.e. the point at which the target word becomes unique from all other words in the language. Other models, like TRACE [10], NAM [5,7] or SHORTLIST [11], which attribute less importance to word onsets, use other definitions of the neighborhood. For instance, NAM is similar to the cohort model in determining initial activation by bottom-up information only (it is also the case in SHORTLIST but not in TRACE), but differs from it in attributing an equal importance to any part of the word. The neighborhood is defined as consisting of all the words that may be generated by the addition, deletion or substitution of a single segment, whatever its position. In TRACE, however, given that new words can begin anywhere in the input stream, competition between candidates can potentially start at different points in time. Whereas this mechanism in TRACE is dealt by multiplicating lexical networks connected by inhibitory links, SORTLIST's main feature is the separation of the recognition process in two successive stages. In the first stage, implemented as a simple recurrent network, all potential lexical candidates beginning at every phoneme in the input are activated in a strictly bottom-up fashion. This "short list" of lexical candidates is then wired into a small interactive network where they compete for recognition.

Another important characteristic of the cohort model is that the activation of neighbors takes place in parallel, at no cost. As a consequence, there should be no effect of the number of neighbors with which the target word has to compete. This feature is in contrast with other models, in particular with NAM. In this model, the probability of recognizing a word should depend both on the word's own frequency and on the number and frequency of its competitors. Neighborhood effects exert their influence by biasing the overall pattern of activity in a decision system as a whole, as opposed to modifying the activation level of a cohort member.

Using each their own definition of the competitor set, Marslen-Wilson [8] found no evidence that the neighborhood density does influence spoken word recognition while Luce [7] reported data suggesting that neighborhood density does influence the process. Regarding neighborhood frequency, Luce found a significant effect in a perceptual identification task (in white noise) and in a lexical decision task. Marslen-Wilson [9], on the other hand, found no effect in lexical decision and in auditory word repetition but an effect on gating isolation points as well as in a cross-modal experiment to which we will return later.

The aim of the present study was to examine the role of neighborhood frequency in spoken word recognition, using 
the neighbors definition of cohort theory. Two sets of spoken words varying by the frequency of their neighbors were compared in three different tasks: lexical decision, auditory repetition and gating.

\section{LEXICAL DECISION}

Method. The participants were 20 undergraduate students who were native speakers of French with no known hearing defects.

The materials consisted of disyllabic CVCV French words of medial frequency and with final uniqueness point: 15 words with at least one more frequent neighbor of the same

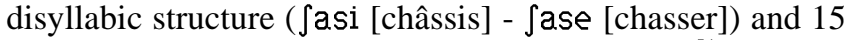
words with no more frequent neighbor (Rybã [ruban). Words from these two sets were matched in frequency and in duration. In order to validate the frequency norms for written words provided by BRULEX [4], a lexical database developed for the French language, a large sample of students were asked to rate the target words and teir potential competitors presented auditorily for their familiarity on a 6 -point scale $(1=$ unknown to $6=$ very frequent). In the set with higher frequency neighbors, the mean subjective frequency estimates obtained for the target words and their neighbors were 4.00 (range: 1.83 - 3.25) and 5.17 (range: $4.58-5.88$ ), respectively. In the set with lower frequency neighbors, they were 4.09 (range: 2.97 4.94 ) and 2.86 (range: 1.41 - 3.82), respectively.

The experiment also included 30 disyllabic CVCV pseudowords derived from the target words by changing the last vowel ( $\left.\int a s \widetilde{\varepsilon}-\mathrm{Rybu}\right)$. All were easily pronounceable. The mean uniqueness point positions from onset and the mean item durations for words and pseudowords appear in Table 1, separately for each neighbors' frequency set.

\begin{tabular}{|c|c|c|c|}
\hline & \multicolumn{2}{|c|}{ Neighbors' frequency } & \multirow[b]{2}{*}{ Higher-Lower } \\
\hline & Lower & Higher & \\
\hline \multicolumn{4}{|l|}{ Words } \\
\hline UP position & 371 & 375 & 4 \\
\hline Duration & 526 & 506 & -20 \\
\hline \multicolumn{4}{|l|}{ Pseudowords } \\
\hline UP position & 368 & 388 & 20 \\
\hline Duration & 493 & 516 & 23 \\
\hline
\end{tabular}

Table 1: Mean uniqueness point (UP) position (ms) and mean item duration (ms) for the different stimulus sets.

Presentation of the items and collection of data were controlled by an Apple IIe computer connected to a DAT converter. The items were recorded on the left channel and a square wave click starting $20 \mathrm{~ms}$ before the onset of each target and triggering a voice key was stored on the right channel. The participants were asked to decide as quickly and accurately as possible by a keypress response whether the target item was a real word or not. Reaction times were measured from stimulus onset.

Results. The mean reaction times and mean percentages or errors for words and pseudowords as a function of the relative frequency of their neighbors are shown in Table 2. These data were submitted to separate Anovas by subject and by item. Reaction times were shorter for words than for pseudowords. This lexicality effect was significant both by subject $\left(F_{\mathrm{S}}(1,19)=81.2 ; \quad p<.005\right)$ and by item $\left(F_{\mathrm{i}}(1,58)=15.4 ; p<.005\right)$. However, the effect of neighborhood frequency reached significance only by subject $\left(F_{\mathrm{S}}(1,19)=5.8 ; p=.025 ; F_{\mathrm{i}}<1\right)$. This slight effect was essentially due to pseudowords $\left(F_{\mathrm{S}}(1,19)=8.9 ; p<.01\right.$; $\left.F_{\mathrm{i}}(1,28)=1.06\right)$, no effect being present for words $\left(F_{\mathrm{S}}\right.$ and $\left.F_{\mathrm{i}}<1\right)$. Actually the small neighborhood frequency effect (25 ms) observed for the pseudoword responses can be interpreted in terms of the difference in item durations, pseudowords with higher frequency neighbors being $23 \mathrm{~ms}$ longer than those with lower frequency neighbors (see Table 1). The analyses run on error rates also failed to reveal any significant effect of neighborhood frequency and lexicality nor interaction.

\begin{tabular}{|llccc|}
\hline \multirow{2}{*}{ Condition } & & $\begin{array}{c}\text { Neighbors' frequency } \\
\text { Lower }\end{array}$ & Higher & Higher-Lower \\
\hline Words & & & & \\
& RT & 815 & 819 & 4 \\
& Errors & 8.3 & 10.3 & 2.0 \\
Pseudowords & & & \\
RT & 877 & 902 & 25 \\
& Errors & 7.3 & 5.0 & -2.3 \\
\hline
\end{tabular}

Table 2: Mean lexical decision reaction times (RTs, ms) and mean error rates (\%) for words and pseudowords as a function of the relative frequency of their neighbors.

Although Luce [7] reported effects of neighborhood frequency on the responses to pseudowords in a lexical decision task, the negative results obtained here corroborate those obtained by Marslen-Wilson [9]. Because the lexical decision task may encourage subjects to wait the end of the stimulus before responding, we decided to use another task, the auditory repetition task, which in principle should not lead to such a strategy.

\section{REPETITION}

Method. The materials used in this experiment and the procedure were the same as in the lexical decision experiment. The participants were 20 undergraduate students different from those of the lexical decision experiment. They were required to repeat the target item as quickly and accurately as possible.

Results. As shown in Table 3, repetition latencies (measured from stimulus onset) tended to be shorter for 
words than for pseudowords $\left(F_{\mathrm{S}}(1,19)=16.1 ; p<.005\right.$; but $\left.F_{\mathrm{i}}(1,56)=1.77 ; p=.19\right)$. There was however no main effect of neighborhood frequency nor interaction between neighborhood frequency and lexicality (all $F$ values $<1$ ). The analyses performed on error rates revealed essentially the same pattern of results, with only a subjects' tendency to produce more errors on words than on pseudowords $\left(F_{\mathrm{S}}(1,19)=10.8 ; p<.005 ;\right.$ but $\left.F_{\mathrm{i}}(1,56)=2.92 ; p=.09\right)$.

\begin{tabular}{|llccc|}
\hline \multirow{2}{*}{ Condition } & \multicolumn{4}{c|}{ Neighbors' } \\
& & Lower & Higher & Higher-Lower \\
\hline Words & & & & \\
& RT & 730 & 714 & -16 \\
& Errors & 1.7 & 2.0 & 0.3 \\
Pseudowords & & & \\
RT & 736 & 745 & 9 \\
& Errors & 5.0 & 3.7 & -1.3 \\
\hline
\end{tabular}

Table 3: Mean repetition reaction times (RTs, ms) and mean error rates (\%) for words and pseudowords as a function of the relative frequency of their neighbors.

Repetition latencies were, on average, $120 \mathrm{~ms}$ shorter than lexical decision times but were still about $200 \mathrm{~ms}$ longer than the mean item duration. These slow reaction times suggest that postperceptual processes are involved, that may hinder some earlier competition effects during lexical selection. Therefore, as Marslen-Wilson did, we decided to run a third experiment using the gating paradigm [6]. This technique does not rule out strategical biases, but can provide some information about early processing.

\section{GATING}

Method. The materials used in this experiment were the same words as in the lexical decision and the repetition experiments (no pseudoword was included). Twenty additional undergraduate students participated as subjects. Each word was presented in several successive fragments of increasing length. The first gate ended at the half of the steady part of the first vowel. The next gates were increased by $30 \mathrm{~ms}$ regular increments. After each presentation, the subject had to write down the word he thought was being presented and to indicate on a 11 point-scale how confident he was about the guess.

Two measures were taken into account : (1) the isolation point, i.e., the average point where the subjects started to guess correctly the target word and did not change their response subsequently and (2) the recognition point, i.e., the average point where the correct response was given a self confidence rate of at least $80 \%$.

Results. The data, which are shown in Table 4, revealed a small effect $(9 \mathrm{~ms})$ of neighborhood frequency on isolation points significant by subject $\left(F_{\mathrm{S}}(1,19)=4.39 ; p=.05\right)$ but not by item $\left(F_{\mathrm{i}}<1\right)$. The effect of neighborhood frequency did not reach significance on recognition points $\left(F_{\mathrm{S}}(1,19)=2.72 ; p=.11 ; F_{\mathrm{i}}<1\right)$. The mean percentages of errors made by the participants at these two average points did not reveal any significant effect of competitor frequency.

\begin{tabular}{|rccc|}
\hline \multirow{2}{*}{ Measure } & \multicolumn{3}{c|}{ Neighbors' frequency } \\
& Lower & Higher & Higher-Lower \\
\hline Isolation points & 297 & 306 & 9 \\
Errors & 1.0 & 2.6 & 1.6 \\
& & & \\
Recognition points & 359 & 364 & 5 \\
Errors & 1.7 & 3.7 & 2.0 \\
\hline
\end{tabular}

Table 4: Mean gating points (in ms from word onset) and mean error rates $(\%)$ as a function of the relative frequency of their neighbors.

The results of this gating experiment thus failed again to show any reliable effect of competitor frequency. They do not confirm those of Marslen-Wilson [9] who reported an effect on isolation points, that is when the sensory information is still insufficient to discriminate between the target word and its competitors.

\section{DISCUSSION}

The present data failed to demonstrate that neighborhood frequency affects spoken word recognition, whatever the task used. Only isolation points in the gating task were slightly affected by neighborhood frequency but the effect was not completely reliable. Before concluding that neighborhood frequency has no or little influence on spoken word recognition, several other possible explanations of these negative results have to be considered.

First, the materials selected in the present experiments were words of medial frequency with or without neighbors of higher relative frequency. This procedure is quite different from that used in other studies [7,9] where low-frequency and high-frequency words had both low-frequency and high-frequency neighbors. It allows also the measurement of relative effects by looking whether item-frequency effects are shifted up or down as a function of the competitor frequency. Because of the larger relative difference in frequency between the target items and their neighbors, the neighborhood frequency effects should in principle be larger than with our procedure. However, this methodological difference may explain reduced effects but probably not their complete abolishment in the present data.

Second, as suggested by Marslen-Wilson [9] who also failed to find any neighborhood frequency effect using the lexical decision and the repetition tasks but a significant effect on gating isolation points, this effect would be a transient phenomenon "that has dissipated by the time the end of the word is reached". In other words, the rapid dissipation of 
activation or inhibition may have prevented the manifestation of any effect with the lexical decision and repetition tasks. Actually, Marslen-Wilson [9] found an effect of competitor frequency in a cross-modal repetition priming experiment, where the subjects had to make a lexical decision on a visual probe immediately preceded by an auditory prime. According to this author, the crucial condition was that the visual probe was presented before the end of the auditory stimulus, when the acoustic information relative to the item being probed and its competitors is still ambiguous. This cross-modal study has however received several criticisms [1]. The effects of neighborhood frequency were measured on visual targets for which only the phonological competitors (not the visual competitors) have been considered. Another difficulty concerns the legitimacy of interpreting effects measured in the visual modality in terms of auditory processes.

Finally, the definition of neighborhood adopted in the present study (in terms of cohort candidates) may be inadequate. The special status attributed by the Cohort model to word onsets has been strongly undermined by number of data obtained with both words excised from fluent speech $[2,12,14]$ and isolated words $[13,15]$. As noted earlier, other current models, whether they incorporate or not the notion of sequential processing, propose different definitions of the neighborhood of a spoken word. The present data do not allow to distinguish between such proposals. What are the competitors of a spoken word and how they exert their influence in the recognition process still remain questions left for future research.

\section{ACKNOWLEDGEMENTS}

This research was supported by the Ministry of Education of the Belgian French-Speaking Community (Concerted Research Action "Language processing in different Modalities: Comparative Approaches") and by the National Fund for Scientific Research (convention 8.4514.95). Monique Radeau is Senior Research Associate of the Belgian Fund for Scientific Research. We thank Christine Eeckman for collecting the data of the gating experiment.

\section{REFERENCES}

[1] Bard, E.G. (1990). Competition, lateral inhibition, and frequency: Comments on the chapters of Frauenfelder and Peeters, Marslen-Wilson, and others. In G.T.M. Altmann (Ed.), Cognitive models of speech processing (pp 185-210). London : MIT Press.

[2] Bard, E. G., Shillcock, R. C., and Altmann, G. T. M. (1988). The recognition of words after their acoustic offsets in spontaneous speech: Effects of subsequent context. Perception and Psychophysics, 44 (5),395408.
[3] Coltheart, M., Davelaar, E., Jonasson, J. T., and Besner, D. (1977). Access to the internal lexicon. In S. Dornic (Ed.), Attention and Performance VI: The psychology of reading (pp. 535-555). New York : Academic Press.

[4] Content, A., Mousty, P., \& Radeau. M. (1990). Brulex: Une base de données lexicales informatisée pour le français écrit et parlé [Brulex: A lexical database for written and spoken French]. L'année Psychologique, 90, 551-566.

[5] Goldinger, S. D., Luce, P. A., \& Pisoni, D. B. (1989). Priming lexical neighbors of spoken words : Effects of competition and inhibition. Journal of Memory and Language, 28, 501-518.

[6] Grosjean, F. (1980). Spoken word-recognition processes and the gating paradigm. Perception and Psychophysics, 28, 267-283.

[7] Luce, P.A. (1986). Neighborhoods of words in the mental lexicon. Doctoral dissertation, Indiana University, Bloomington, Indiana.

[8] Marslen-Wilson, W. D. (1987). Functional parallelism in spoken word-recognition. Cognition, 25, 7-102.

[9] Marslen-Wilson, W. D., (1990). Activation, competition, and frequency in lexical access. In G.T.M. Altmann (Ed.), Cognitive models of speech processing (pp 148-172). London: MIT Press.

[10] McClelland, J. L., and Elman, J.L. (1986). The TRACE model of speech perception. Cognitive Psychology, 18, 1-86.

[11] Norris, D. (1994). Shortlist: A connectionist model of continuous speech recognition. Cognition, 52, 189-234.

[12] Pickett, J. M., \& Pollack, I. (1963). Intelligibility of excerpts from fluent speech: Effects of rate of utterance and duration of excerpt. Language and speech, 6, 151-164.

[13] Radeau, M., Mousty, P., \& Pasdeloup, V. (1994). L'effet du débit de parole sur le rôle du point d'unicite [The effect of articulation rate on the role of the uniqueness point]. Actes des XXèmes Journées d'Etude sur la Parole, Trégastel, June 1-3, 1994.

[14] Schillcock, R.C. (1990). Lexical hypotheses in continuous speech. In G.T.M. Altmann (Ed.), Cognitive models of speech processing ( $\mathrm{pp}$ 24-49). London: MIT Press.

[15] Taft, M, \& Hambly, G. (1986). Exploring the cohort model of spoken word recognition. Cognition, 22, 259-282. 